\title{
Millimeter wave spectroscopic measurements of stratospheric and mesospheric constituents over the Italian Alps: stratospheric ozone
}

\author{
Giovanni Muscari $\left({ }^{1}\right)$, Claudio Cesaroni $\left({ }^{1}\right)$, Cesidio Bianchi $\left({ }^{1}\right)$, Robert L. de Zafra $\left({ }^{2}\right)$, Tatiana Di Iorio $\left({ }^{3}\right)$, \\ Irene Fiorucci $\left({ }^{1}\right)$, Daniele Fuà $\left({ }^{3}\right)$, Vito Romaniello $\left({ }^{3}\right)$ and Enrico Zuccheretti $\left({ }^{1}\right)$ \\ $\left({ }^{1}\right)$ Istituto Nazionale di Geofisica e Vulcanologia, Roma, Italy \\ (') Department of Physics and Astronomy and Institute for Terrestrial and Planetary Atmospheres, \\ State University of New York, Stony Brook, U.S.A. \\ $\left({ }^{3}\right)$ Dipartimento di Fisica, Università degli Studi di Roma «La Sapienza», Roma, Italy
}

\begin{abstract}
Measurements of rotational lines emitted by middle atmospheric trace gases have been carried out from the Alpine station of Testa Grigia $\left(45.9^{\circ} \mathrm{N}, 7.7^{\circ} \mathrm{E}\right.$, elev. $\left.3500 \mathrm{~m}\right)$ by means of a Ground-Based Millimeter-wave Spectrometer (GBMS). Observations of species such as $\mathrm{O}_{3}, \mathrm{HNO}_{3}, \mathrm{CO}, \mathrm{N}_{2} \mathrm{O}, \mathrm{HCN}$, and $\mathrm{HDO}$ took place during 4 winter periods, from February 2004 to March 2007, for a total of 116 days of measurements grouped in about 18 field campaigns. By studying the pressure-broadened shape of emission lines the vertical distribution of the observed constituents is retrieved within an altitude range of $\sim 17-75 \mathrm{~km}$, constrained by the $600 \mathrm{MHz}$ pass band and the $65 \mathrm{kHz}$ spectral resolution of the back-end spectrometer. This work discusses the behavior of stratospheric $\mathrm{O}_{3}$ during the entire period of operation at Testa Grigia. Mid-latitude $\mathrm{O}_{3}$ columnar content as estimated using GBMS measurements can vary by large amounts over a period of very few days, with the largest variations observed in December 2005, February 2006, and March 2006, confirming that the northern winter of 2005-2006 was characterized by a particularly intense planetary wave activity. The largest rapid variation from maximum to minimum $\mathrm{O}_{3}$ column values over Testa Grigia took place in December 2006 and reached a relative value of $72 \%$ with respect to the average column content for that period. During most GBMS observation times much of the variability is concentrated in the column below $20 \mathrm{~km}$, with tropospheric weather systems and advection of tropical tropospheric air into the lower stratosphere over Testa Grigia having a large impact on the observed variations in column contents. Nonetheless, a wide variability is also found in middle stratospheric GBMS $\mathrm{O}_{3}$ measurements, as expected for mid-latitude ozone. We find that $\mathrm{O}_{3}$ mixing ratios at $\sim 32 \mathrm{~km}$ are very well correlated with the solar illumination experienced by air masses over the previous $\sim 15$ days, showing that already at $32 \mathrm{~km}$ altitude ozone photochemistry dominates over transport processes. The correlation of lower stratospheric ozone concentrations with potential vorticity as an indicator of transport is instead not as clear-cut, due to very complex mixing processes that characterize stratospheric air at mid-latitudes. Correlations of $\mathrm{O}_{3}$ over Testa Grigia with stratospheric tracers such as $\mathrm{N}_{2} \mathrm{O}$ and $\mathrm{HCN}$, also observed by means of the GBMS, are planned for the future, in order to better characterize lower stratospheric dynamics and therefore lower stratospheric ozone concentrations at mid-latitudes.
\end{abstract}

Key words millimeter-wave spectroscopy - stratospheric ozone - isentropic transport

Mailing address: Dr. Giovanni Muscari, Istituto Nazionale di Geofisica e Vulcanologia, Via di Vigna Murata 605, 00143 Roma, Italy; e-mail: muscari@ingv.it

\section{Introduction}

From February 2004 to March 2007 a collaboration among the University of Rome «La Sapienza», the State University of New York at Stony Brook (SUNYSB), and the Istituto Nazio- 
nale di Geofisica e Vulcanologia, with the logistic support of the Istituto di Fisica dello Spazio Interplanetario (INAF/IFSI), operated a ground-based millimeter-wave spectrometer (GBMS) measuring middle atmospheric trace gases from the Alpine site of Testa Grigia (or Plateau Rosa, $45.9^{\circ} \mathrm{N}, 7.7^{\circ} \mathrm{E}$, elev. $3500 \mathrm{~m}$ above mean sea level), at the border between Italy and Switzerland. The high elevation makes Testa Grigia (hereafter referred to as TG) an excellent site for carrying out atmospheric or astronomic measurements at mid-latitudes with instruments, such as the GBMS, which operate at frequencies where atmospheric transparency depends heavily on water vapor and therefore a low water vapor columnar content is necessary. Daily spectroscopic measurements were carried out during several field campaigns mostly during fall and winter, when water vapor columnar content (or precipitable water vapor, PWV) is the lowest. The GBMS observed rotational emission lines of $\mathrm{O}_{3}$ at 276.923 $\mathrm{GHz}, \mathrm{HNO}_{3}$ at $269.211 \mathrm{GHz}, \mathrm{CO}$ at 230.538 $\mathrm{GHz}, \mathrm{N}_{2} \mathrm{O}$ at $276.328 \mathrm{GHz}, \mathrm{HCN}$ at 265.886 $\mathrm{GHz}$, and $\mathrm{HDO}$ at $255.050 \mathrm{GHz}$. The pressurebroadened spectral line shapes allow retrieval of the vertical distribution of the emitting trace gases between approximately 17 and $75 \mathrm{~km}$ (see Section 2 for details).

This work discusses the behavior of stratospheric $\mathrm{O}_{3}$ observed during a total of 116 days over 4 winters, grouped in about 18 field campaigns. At mid-latitudes total ozone is controlled by both transport and chemistry. Ozone forms mainly in the tropical stratosphere and is advected poleward leading to an increasing total ozone column at higher latitudes during winter and spring. At mid-latitudes stratospheric ozone is characterized by a considerable seasonal variability with lowest values in autumn and a strong variability on time scales from days to months which leads to variations in total ozone that can exceed $50 \%$ of average values. Events with very low and very high total ozone values are often called miniholes and minihighs, respectively (e.g., Koch et al., 2005; Stick et al., 2006), and are identified as synoptic-scale regions characterized by total column ozone values significantly below/above a certain threshold.

Ozone miniholes are primarily the result of dynamic-atmospheric processes, given that they evolve too rapidly to be the result of an ozone chemical destruction mechanism (Koch et al., 2005). The dynamic origin of such extreme ozone events was already noted by Dobson et al. (1929), who described an interrelation between fluctuations in total ozone and the passing of weather systems. Currently, the exact dynamical mechanisms of their formation are under dispute, with two main hypotheses: i) the buildup of an intense tropospheric high-pressure system with an elevated tropopause and the subsequent generation of a reduced ozone layer thickness (Teitelbaum et al., 2003); ii) the generation of an air column with low ozone mixing ratios caused by meridional isentropic transport of air from the subtropics between 340 and $440 \mathrm{~K}(190-70 \mathrm{hPa})$ and, independently, from polar regions between 530 and $700 \mathrm{~K}(30-15 \mathrm{hPa})$, both carrying air with climatologically low ozone mixing ratios (Koch et al., 2002). Koch et al. (2005) studied the relative importance of the two mechanisms, quantified statistically for all miniholes and minihighs observed over Payerne, Switzerland, in the time period 1980-1989 and concluded that fast isentropic transport is by far the most important process for minihole genesis.

From a study by Hood et al. (2001) on 71 miniholes between 1980 and 1993, ozone loss at polar latitudes caused by heterogeneous chemistry due to the presence of Polar Stratospheric Clouds (PSCs) was estimated to contribute less than $1 \%$ to the observed low column ozone at mid-latitudes, although more recently a PSC and a conspicuous related chemical ozone depletion was observed over Southern Europe in January 2006 (Keckhut et al., 2007; Keil et al., 2007).

\section{Observing technique and profile retrieval}

The GBMS is currently a state-of-the-art system that employs a Superconductor-Insulator-Superconductor (SIS) tunnel junction mixer to convert millimeter wave signals arising from molecular rotational transitions to lower «Intemediate» Frequencies (IF) by heterodyning them with a local oscillator. The IF output is fed to a «wide band» Acousto-Optical Spectrometer (AOS) characterized by a band pass of 600 $\mathrm{MHz}$ and a spectral resolution of $1.2 \mathrm{MHz}$, and 
to a «narrow band» AOS with a $50 \mathrm{MHz}$ band pass and $65 \mathrm{kHz}$ of resolution. The $50 \mathrm{MHz}$ window of the narrow band AOS is adjusted within the $600 \mathrm{MHz}$ band pass of the wide band AOS in order to gain a higher resolution of spectral line centers associated with high altitude emission.

Our GBMS system is employed to measure molecular rotational lines of several trace gases that emit in the frequency range between 230 and $280 \mathrm{GHz}$. Since spectral line shapes are determined by a convolution of the vertical concentration profile (typically unknown) and the atmospheric pressure profile (typically known), the latter can be used, along with the observed line shape, in a mathematical deconvolution process to find the emitting molecule's concentration as a function of altitude. The overall spectral band pass and resolution of the GBMS are therefore key elements to determine the altitude range where trace gases concentration can be measured. Assuming a generic line pressure broadening of $\sim 3 \mathrm{MHz} / \mathrm{mbar}$, with the aid of pressure and temperature vertical profiles the GBMS allows the retrieval of vertical distributions of trace gases concentration between $\sim 17$ and $\sim 70-75 \mathrm{~km}$ altitude. Above $70-75 \mathrm{~km}$, pressure broadening is overcome by Doppler broadening, and only the overlying column density can be determined with confidence. GBMS measurements are integrated over time intervals between $10 \mathrm{~min}$ and several hours, depending on the intensity and therefore the signal to noise ratio of the spectral line being observed.

In this work we discuss measurements of the pure rotational transition line of $\mathrm{O}_{3}$ at 276.923 $\mathrm{GHz}$. This line is strong enough to give signalto-noise ratios at line center larger than 100:1 in a 10 to $20 \mathrm{~min}$ integration, though still weak enough to show relatively little self-absorption by lower levels for radiation originating in upper layers of the stratosphere. Spectra have been processed with the Chahine-Twomey deconvolution technique (Twomey et al., 1977) to retrieve vertical profiles from the measured line shapes. The wing of a second, strong ozone line centered at $274.478 \mathrm{GHz}$, outside the spectral window, contributes background curvature and is subtracted from the measured spectra in the data pre-processing phase.
The inversion procedure starts by synthesizing the $\mathrm{O}_{3}$ line that would be generated by an initial $\mathrm{O}_{3}$ volume mixing ratio (vmr) vertical profile, knowing the physical characteristics of the rotational transition observed. The modeled spectrum is then compared to the measured one and the initial vmr profile is modified iteratively until the differences between computed and measured spectral line shapes are minimized. Changes to the initial $\mathrm{O}_{3}$ vertical profile are generated that are based on weighting functions (or averaging kernels) which relate the shape of the $\mathrm{O}_{3}$ rotational line over a given frequency range (measured from the line center) to the concentration of $\mathrm{O}_{3}$ over a specific altitude range. The $\mathrm{O}_{3}$ vmr vertical profile cannot be retrieved below altitudes where the pressure broadening is much greater than the total spectral bandwidth. The GBMS's $600 \mathrm{MHz}$ spectral window thus limits the bottom altitude level of our retrievals to $\sim 15$ $17 \mathrm{~km}$ altitude. In practice, GBMS $\mathrm{O}_{3}$ retrievals are computed down to ground level, but they rely almost entirely on initial profiles below $\sim 15$ $\mathrm{km}$ altitude (see next paragraph).

Deconvolutions were performed using several choices of starting $\mathrm{O}_{3} \mathrm{vmr}$ vertical profiles and weighting functions to minimize any biasing from a particular set of inputs, and the recovered vertical profiles were averaged. Initial profiles below $20 \mathrm{~km}$ altitude were computed using ozone sonde measurements carried out by MeteoSwiss from Payerne (e.g., Jeannet et al., 2007), Switzerland, about $100 \mathrm{~km}$ away from TG. Atmospheric pressure and temperature profiles used in the analysis were obtained from the National Center for Environmental Prediction (NCEP) for appropriate dates and location. Ozone mixing ratios are retrieved every $\mathrm{km}$, although due to the finite width of the millimeter-wave averaging kernels (i.e., the radiation measured at a frequency separated $\Delta v$ from the line center depends on $\mathrm{O}_{3}$ molecules emitting from a wide range of altitudes, not a very thin layer), uncorrelated information is obtained at layers separated by $\sim 6 \mathrm{~km}$ in the lower to mid-stratosphere, increasing to more than 10 $\mathrm{km}$ in the upper stratosphere, determined by the Full Width at Half-Maximum (FWHM) of the averaging kernels used in the inversion algorithm. Figure 1 gives an illustration of the spectral line shape quality, after removal of minor background 

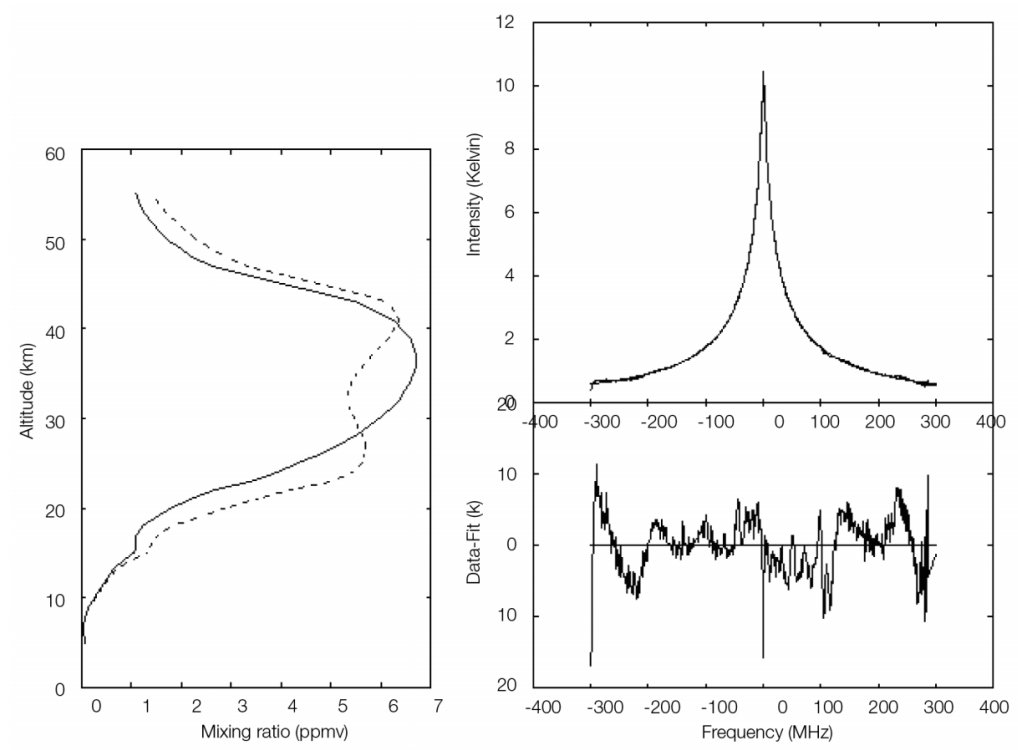

Fig. 1. (Top, right) Measured spectral line data after subtraction of computed background from other nearby ozone transitions. A line shape synthesized from the retrieved vertical profile (solid line, left panel) is superimposed on the observed spectrum, but can be barely distinguished. The difference spectrum between these is shown on an enlarged scale (bottom, right). Spectral intensity is given in kelvins for the equivalent emission temperature in the Rayleigh-Jeans approximation. The dashed line in the left hand panel is one of three starting profiles used for deconvolution of each day's data. The solid line is the profile retrieved from the data in the upper right panel.

contributions from other ozone emission, along with a typical starting profile, the retrieved vmr profile, and the residual between the observed line shape and a line shape synthesized from the retrieved profile.

Uncertainties on GBMS $\mathrm{O}_{3}$ vmr vertical profiles have several sources. The most important contributions are: background removal process; choice of starting profiles and averaging kernels; input parameters such as transition line intensity, molecular partition function, pressure-broadening coefficient; random noise in spectral data; calibration, i.e. the radiometric conversion from voltages to radiances; and evaluation of the tropospheric attenuation of stratospheric signals. Assuming that the most important contributions to the overall uncertainty are independent from one another, we added them in quadrature and obtained an overall $\pm 13 \%$ uncertainty on GBMS $\mathrm{O}_{3}$ vmr values.

The millimeter-wave emission spectroscopy employed by the GBMS has several advantages over other techniques for measuring atmospheric constituents. With respect to balloonsonde data, GBMS vertical profiles of chemical compounds reach much higher altitudes (a maximum altitude of $70-75 \mathrm{~km}$ instead of $\leq 35 \mathrm{~km}$ ), although they have much lower vertical resolution. Once the millimeter-wave receiver is set up, measurements can be carried out daily over several months, and at relatively low cost, by a single operator. Furthermore, balloon sondes typically carry out measurements of one single chemical species on each flight, (unless the sonde carries expensive instrumentation that will need to be recovered after the flight, with the risk of being lost), while the GBMS can be rapidly tuned to different frequencies and measure the emission from several species within $24 \mathrm{~h}$.

With respect to remote sensing techniques at infrared (IR) or visible wavelengths, millimeterwave spectroscopy is able to exploit the pressure broadening characteristics of spectral lines, because this is much larger than Doppler broaden- 
ing at the long wavelengths and correspondingly lower frequencies employed. The measure of pressure broadening allows the retrieval of vertical profiles, which with rare exceptions cannot be obtained by ground-based IR and visible techniques, so that the latter are primarily capable of measuring only columnar content. IR and visible spectroscopy are able to produce reliable vertical profiles when instruments are used in a limbsounding mode onboard satellites or very high altitude airplanes, but such experiments are orders of magnitude more expensive than groundbased techniques, and typically have a much shorter operational duty cycle. Optical spectroscopy from any platform is also limited to daylight hours and thus unsuitable for species which may only form during the night.

The GBMS was deployed mostly to polar regions: to the Antarctic Amundsen-Scott base $\left(90^{\circ} \mathrm{S}\right)$ during 1993, 1995, and 1999 (e.g., Cheng et al., 1996; de Zafra et al., 1997), and to Thule $\left(76.5^{\circ} \mathrm{N}, 68.7^{\circ} \mathrm{W}\right)$, Greenland, during the winters of 2001-2002 and 2002-2003 (de Zafra and Muscari, 2004; Muscari et al., 2007). The overall design and details of the observing technique have been discussed at length by de Zafra (1995) and Parrish et al. (1988), while a summary on the accuracy of the inversion procedures used to deconvolve the different spectral lines can be found in Muscari et al. (2007) and in references therein.

\section{Results and discussion}

Figure 2 shows $\mathrm{O}_{3}$ column contents derived from GBMS vertical profiles from the ground to $50 \mathrm{~km}$ (contribution from higher altitudes is negligible), with larger solid symbols representing the average value for each group of data (i.e., field campaign, with its first and last day of measurements indicated at the top of the corresponding panel in fig. 5, discussed later). Below $15 \mathrm{~km}$ GBMS vertical profiles, and therefore column contents, rely significantly on ozone sonde measurements from Payerne, as discussed in Section 2. A weighted average of the uncertainty contributions from ozone sonde and GBMS to the final $\mathrm{O}_{3}$ column contents provides an estimate for the total uncertainty of $\pm 12 \%$ (Cheng et al., 1996).

In figure 2 we notice that $\mathrm{O}_{3}$ columnar contents measured within a few days can be scattered over a wide range of values, with the largest vari-

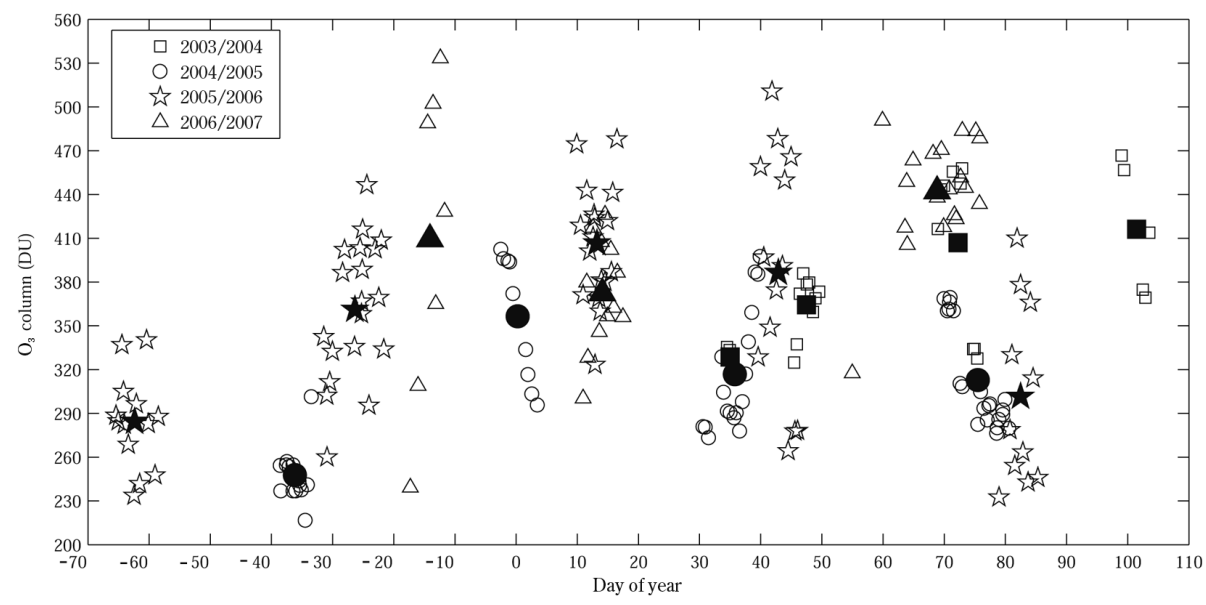

Fig. 2. GBMS O3 total column density (in Dobson Units) versus day of the year for all 4 winter periods, with 31 December at 12 GMT as day -0.5 and 1 January as day 1 . The legend shows which symbol represents each winter. Solid symbols are average values for each field campaign with first and last day of each campaign indicated in fig. 5. 
ations observed in December 2005 (52\% of the campaign average value indicated with a solid star), February 2006 (64\% of the campaign average value indicated with a solid star), March 2006 (59\% of the campaign average value indicated with a solid star) and December 2006 (72\% of the campaign average value indicated with a solid triangle). Interestingly, in most periods a large variability is concentrated in the column below 20 $\mathrm{km}$ (not shown), suggesting that tropospheric weather systems and advection of tropical tropospheric air into the lower stratosphere over TG has a large impact on the observed variations in col- umn contents. Furthermore, out of the 4 mentioned periods of particularly large $\mathrm{O}_{3}$ column variability, 3 took place during winter 2005-2006, which proved to be a period of large planetary wave activity, starting with a Canadian Warming and the intensification of the Aleutian high pressure system at the end of November (Braathen, 2006), just before the December 2005 GBMS field campaign. Afterwards, several more perturbations took place until on 21 January a major midwinter warming [which is considered to happen when at $10 \mathrm{hPa}$ and above the temperature difference between the pole and $60^{\circ} \mathrm{N}$ is reversed

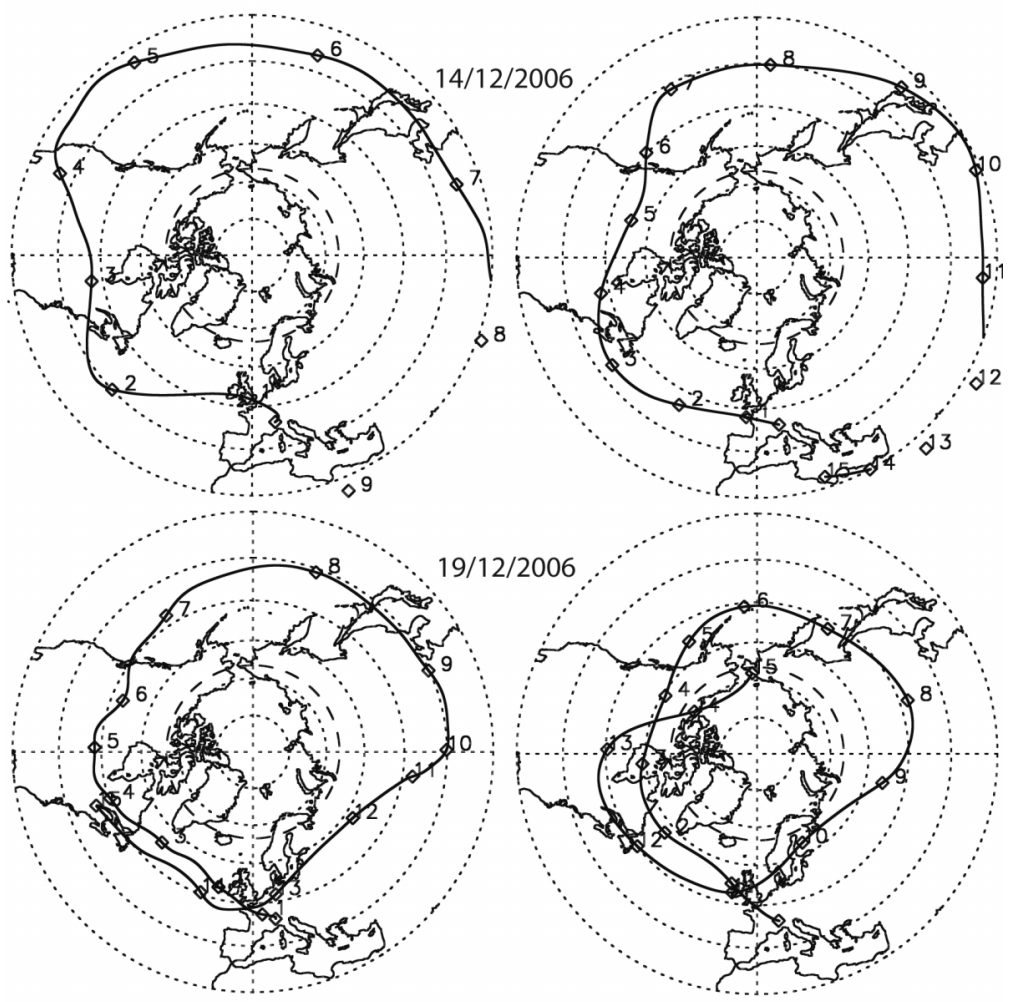

Fig. 3. 15-day back trajectories at $350 \mathrm{~K}(\sim 13 \mathrm{~km}$ altitude; top and bottom left $)$ and $430 \mathrm{~K}(\sim 18 \mathrm{~km}$ altitude; top and bottom right) ending over Testa Grigia on 14 December (top) and 19 December (bottom), 2006. For each of the four plots, five trajectories were calculated and only the resulting average trajectory is shown (see text for details). Numbers along each trajectory indicate the distance in days before the arrival of the air parcel at Testa Grigia. Trajectories are not drawn when they pass below $30^{\circ} \mathrm{N}$. Back trajectory calculations are from the NASA/Goddard Space Flight Center (NASA/GSFC) «automailer» system initiated with NCEP reanalysis meteorological data (Schoeberl and Sparling, 1994). 


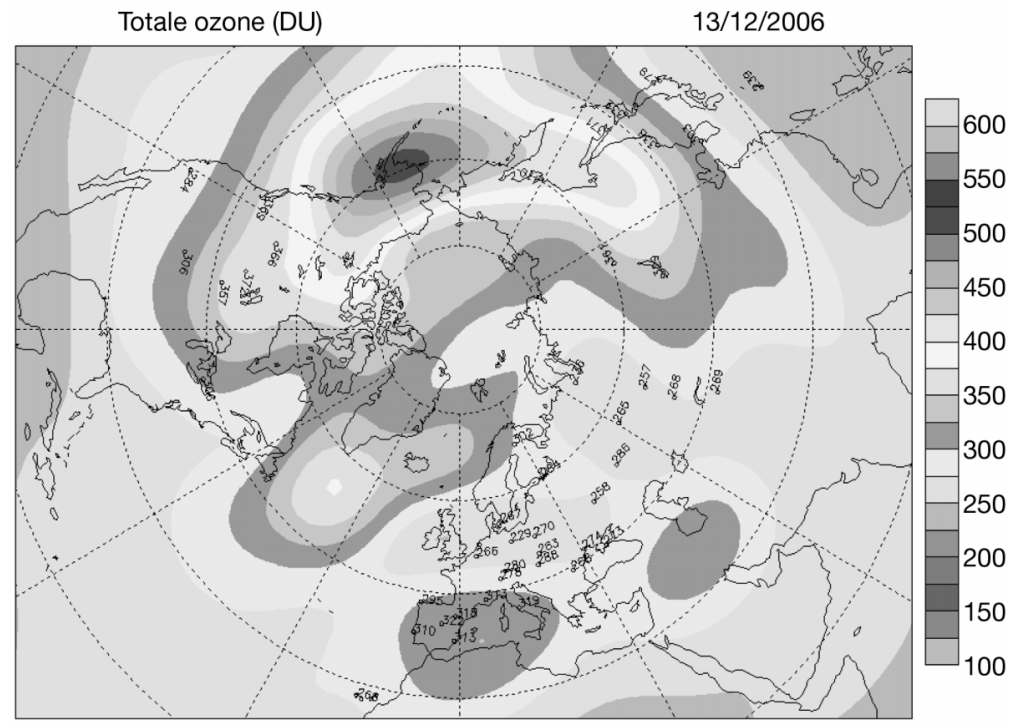

Fig. 4. Ozone total column contour map in Dobson Units from the World Ozone and Ultraviolet Radiation Data Centre (WOUDC) in Toronto (<http://es-ee.tor.ec.gc.ca>) for 13 December, 2006.

(positive) and the mean zonal wind at $60^{\circ} \mathrm{N}$ reaches negative values (easterlies)] occurred in the Arctic stratosphere. This is considered one of the strongest and most prolonged sudden warmings on record, lasting for about 4 weeks (Manney et al., 2007) and altered $\mathrm{O}_{3}$ concentrations at mid-latitudes.

As for December 2006 (empty triangles in fig. 2), we examined in more details stratospheric dynamics between days 14 to 19 of December, when the GBMS observed the minimum and maximum column contents, respectively. The low ozone contents measured by the GBMS on 14 December seems to be caused by air masses that reached the lower stratosphere above TG originating from the ozone deprived upper tropical troposphere, as suggested by isentropic air parcel trajectories advected back 15 days at 350 and $430 \mathrm{~K}$ of potential temperature ( $\sim 13$ and $18 \mathrm{~km}$, respectively) and shown in fig. 3. For each of the four plots shown in the figure, five trajectories were calculated and only the resulting average trajectory is shown. One of the five trajectories ends exactly at Testa Grigia while the other four end at locations surrounding Testa Grigia at four cardinal points sep- arated by $90^{\circ}$. This effort aimed at gaining more confidence in the history of air masses passing over TG by observing the behavior of a larger volume of air with respect to the volume corresponding to one location only. Back trajectory calculations are from the NASA/Goddard Space Flight Center (NASA/GSFC) «automailer» system initiated with NCEP reanalysis meteorological data (Schoeberl and Sparling, 1995). Large $\mathrm{O}_{3}$ values observed on 18 and 19 December can instead be explained by the advection over TG of stratospheric air rich in $\mathrm{O}_{3}$ from the Aleutian region, as suggested by the dynamics of air parcels that 7 days before their passage over TG were found over the high ozone region. This conclusion is illustrated by the combination of air trajectories in fig. 3 and the $\mathrm{O}_{3}$ map provided by the World Ozone and Ultraviolet Radiation Data Centre (WOUDC) in Toronto (<http://es-ee.tor.ec.gc.ca $>$ ) for 13 December, shown in fig. 4.

Throughout the field campaigns that show large $\mathrm{O}_{3}$ column variability, $\mathrm{O}_{3}$ total column values provided by the WOUDC and by the Ozone Monitoring Instrument (OMI) onboard the EOS AURA satellite (<http://acdisc.sci.gsfc.nasa.gov>) 
Giovanni Muscari et al.
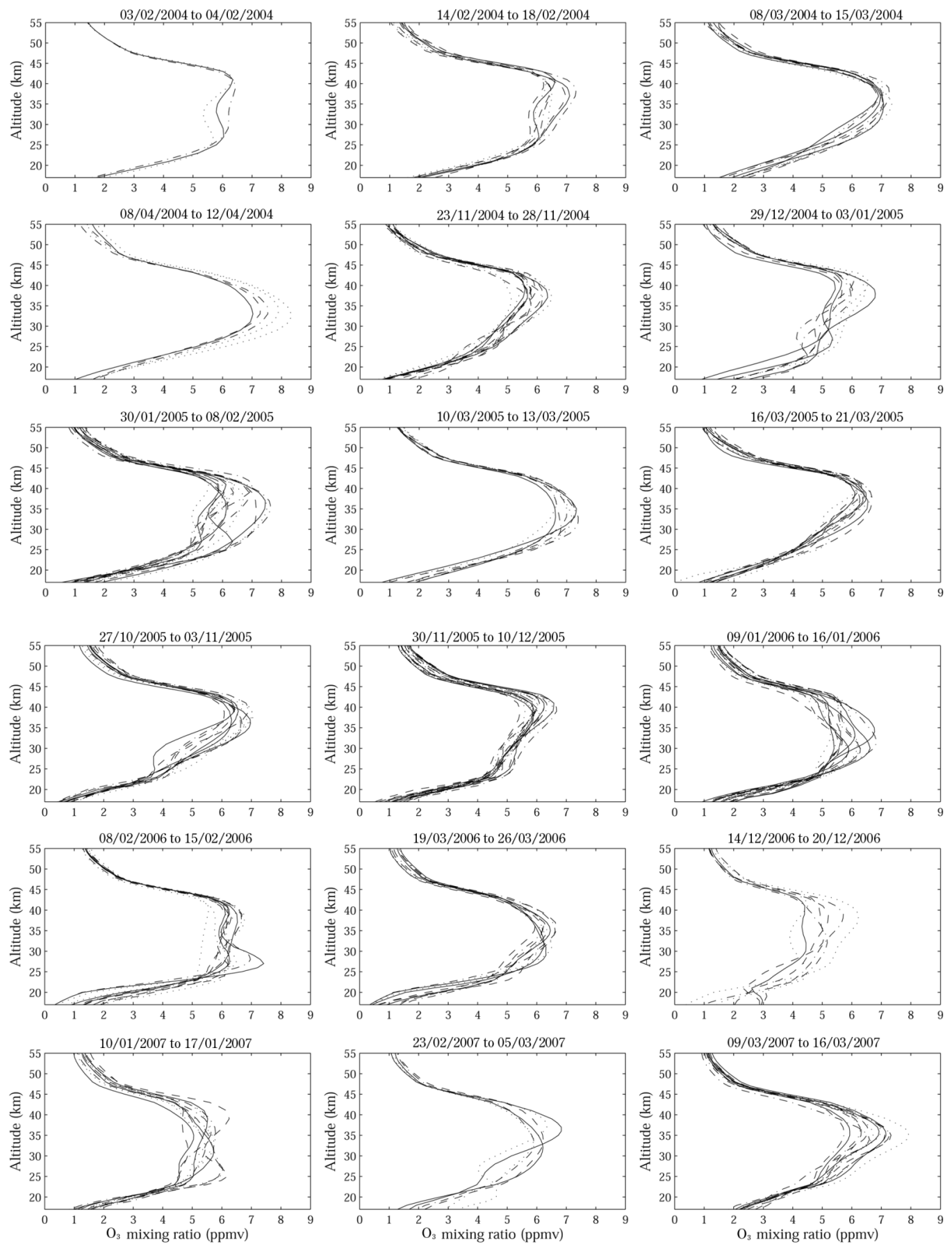

Fig. 5. GBMS $\mathrm{O}_{3}$ mixing ratio vertical profiles from 17 to $55 \mathrm{~km}$ altitude from Testa Grigia. Each panel depicts measurements from one field campaign, with first and last day of measurements indicated at the top of each panel. 
confirmed qualitatively the large variations observed by the GBMS over TG. In particular, for all the extreme events (very large or very little $\mathrm{O}_{3}$ content) observed by the GBMS we found a good correspondence in timing (i.e., days of occurrence) with maxima and minima $\mathrm{O}_{3}$ total column values over TG found in WOUDC and OMI/AURA databases, although the latter dataset has a coarser horizontal resolution and therefore shows smaller variations with respect to those observed by the GBMS.

Column contents are most sensitive to changes in $\mathrm{O}_{3}$ concentrations in the lower stratosphere. In order to discuss observations of middle stratospheric $\mathrm{O}_{3}$ all the vertical profiles obtained from GBMS field campaigns at TG are displayed in fig. 5. Figure panels depict measurements obtained during each campaign, with their first and last day of measurements indicated at the top of each panel. The same data set is shown in fig. 6 by means of contour maps in order to better illustrate the time periods covered by GBMS measurements.

Ozone vmr vertical profiles confirm the large degree of variability over short time spans observed for column contents and characteristic of mid-latitude ozone concentrations. Interesting features are the double peaked structure observed in several profiles and usually addressed with the term «low ozone pocket» identified by the decrease in ozone mixing ratio between 30 and $35 \mathrm{~km}$ altitude and usually characteristic of air from polar regions during winter, when air can remain isolated in the dark for several days and reaches a different photochemical equilibrium (towards lower ozone values) with respect to mid-latitude and tropical air (e.g., Cheng et al., 1996; Muscari et al., 2007, and references therein). Since ozone sonde measurements barely reach $30 \mathrm{~km}$ altitude, the large set of ozone sondes launched at mid-latitudes completely miss this feature in $\mathrm{O}_{3}$ mixing ratio profiles. Low ozone pockets are not as deep at mid-latitudes as they are over the Antarctic continent during winter (Cheng et al., 1996). However, just as it can be shown from observations at polar latitudes (Muscari et al., 2007), also at mid-latitudes there is a strong correlation between ozone mixing ratio at $\sim 32 \mathrm{~km}$ and the illumination fraction (defined as the ratio between the time interval dur- ing which the air parcel received solar illumination and the total duration of the trajectory) experienced by air parcels in the preceding 10 days. Figure 7 displays such a correlation at a potential temperature of $850 \mathrm{~K}(\sim 32 \mathrm{~km})$. The entire GBMS $\mathrm{O}_{3}$ data set from TG is shown with solid circles, the GBMS data set from the 2002 field campaign at Thule $\left(76.5^{\circ} \mathrm{N}, 68.7^{\circ} \mathrm{W}\right)$, Greenland, is shown with open squares (see also Muscari et al., 2007), and stars superimposed to open squares are measurements at TG during 18-21 March, 2005.

The correlation of the two datasets with illumination fraction is strong, with correlation coefficients of 0.66 and 0.82 for TG and Thule data, respectively, demonstrating that already at $32 \mathrm{~km}$ altitude the ozone photochemical lifetime is comparable to transport time scales, i.e., ozone concentrations are driven as much by photochemistry as by transport. The offset that separates the two correlations, indicated by the two different linear fits (dotted and solid lines for TG and Thule, respectively) in fig. 7 is possibly due to the different history (preceding the 10 -day period examined with the use of air trajectories) of the air masses sampled in the two regions. This includes the exposure of the two sets of air masses to atmospheric physical properties, e.g., diabatic descent or average temperature, that can be very different at polar versus mid-latitudes. Air masses sampled in the period 18-21 March, 2005 (stars overlaid on empty squares in fig. 7), belong to the inner polar vortex and arrived from the polar vortex to TG very rapidly, as shown by air parcel trajectories depicted in fig. 8. In fact, during this period, at potential temperatures between $550 \mathrm{~K}$ and at least $1000 \mathrm{~K}$ ( 24-34 km altitude) Potential Vorticity (PV) maps (not shown) prove that the polar vortex shifted over Central Europe and lay directly over TG. The corresponding data points in fig. 7 fit very well with the linear trend for Thule 2002 data. Only about $50 \%$ of the remaining outliers in the $\mathrm{TG} \mathrm{O}_{3}$ /illumination fraction correlation (that agree well with the correlation from Thule) can be clearly explained as characteristic of air rapidly advected from the Arctic region to mid-latitudes. During this same period, fig. 2 shows consistently low $\mathrm{O}_{3}$ column contents (empty circles) with respect to season 


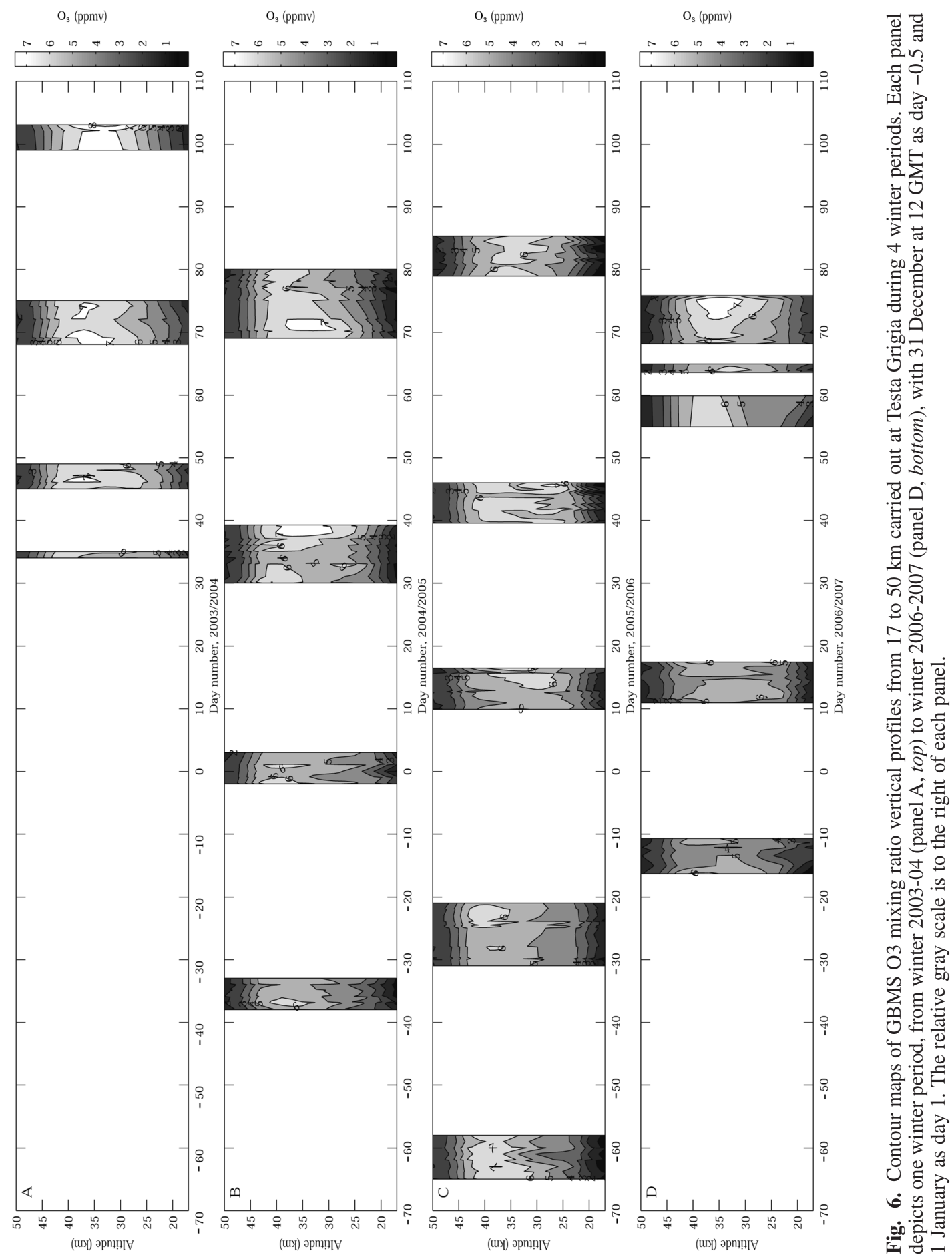




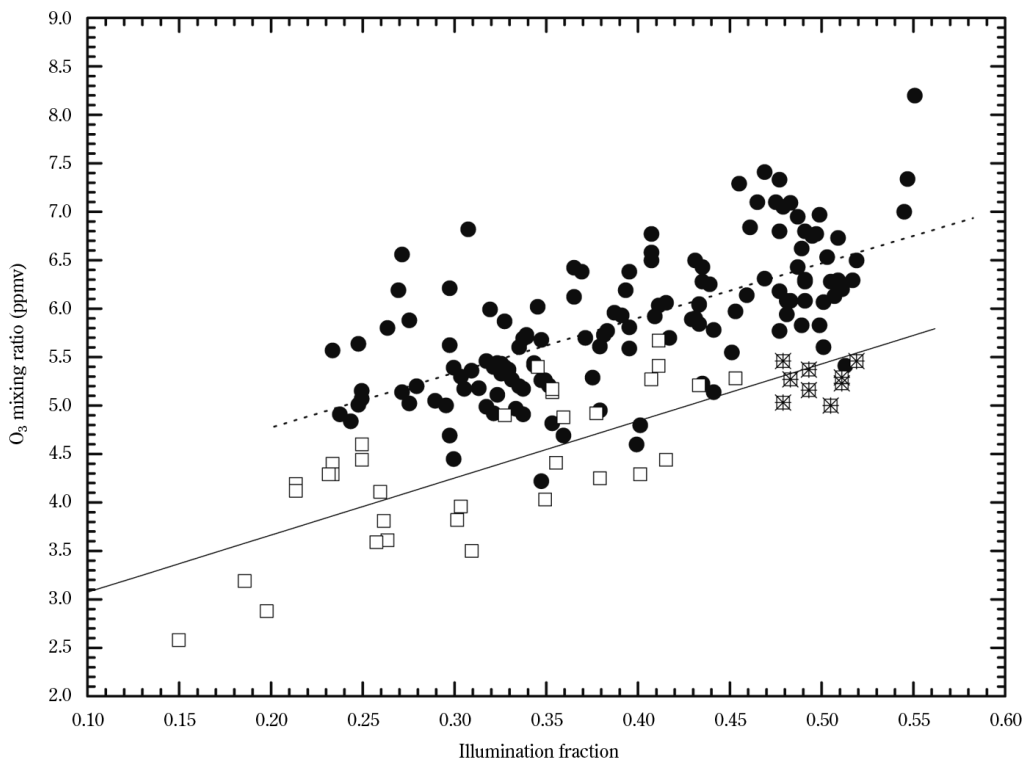

Fig. 7. Correlation scatterplot between all the Testa Grigia GBMS ozone mixing ratio values at $850 \mathrm{~K}$ and illumination fraction values along 15-day back trajectories at $850 \mathrm{~K}$ (solid circles), and between the same quantities but from the winter 2001-2002 Thule field campaign (open squares; see also Muscari et al., 2007). Stars superimposed to open squares are measurements from 18-21 March, 2005 (see text for details). Dotted and solid lines are linear fits to Testa Grigia and Thule data, respectively. The illumination fraction is defined as the ratio between the time interval during which the air parcel received solar illumination and the total duration of the trajectory. The correlation coefficients are 0.66 and 0.82 for Testa Grigia and Thule data, respectively.

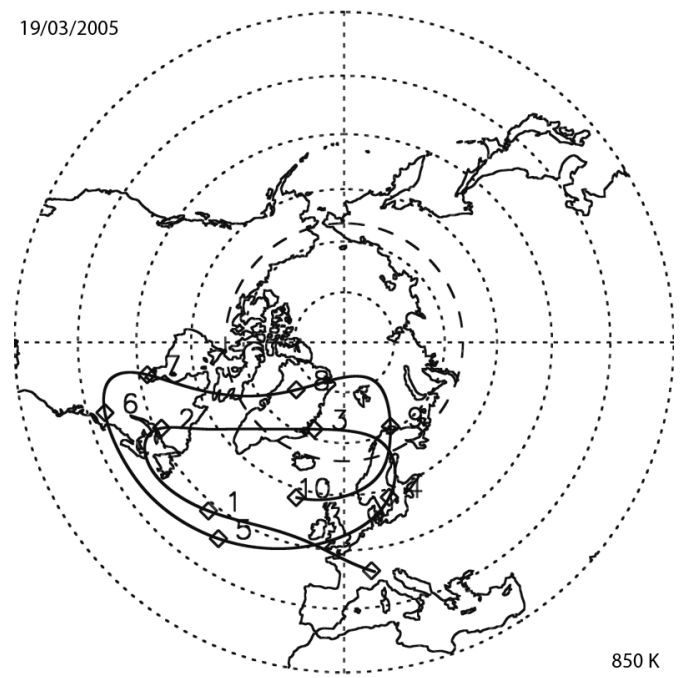

Fig. 8. 10-day NASA/GSFC back trajectory at 850 $\mathrm{K}$ arriving over Testa Grigia on 19 March, 2005. Five trajectories were calculated and only the resulting average trajectory is shown (see text for details). Numbers along the trajectory indicate the distance in days before the arrival of the air parcel at Testa Grigia. Back trajectory calculations are from the NASA/Goddard Space Flight Center (NASA/GSFC) «automailer» system initiated with NCEP reanalysis meteorological data (Schoeberl and Sparling, 1994).

averages, in agreement with OMI/AURA and WOUDC datasets. This is due to the advection over TG of polar vortex air also at altitudes lower than the $850 \mathrm{~K}$ isentropic surface.

Figure 9 displays the correlation between GBMS column values of $\mathrm{O}_{3}$ from 24 to $29 \mathrm{~km}$ 


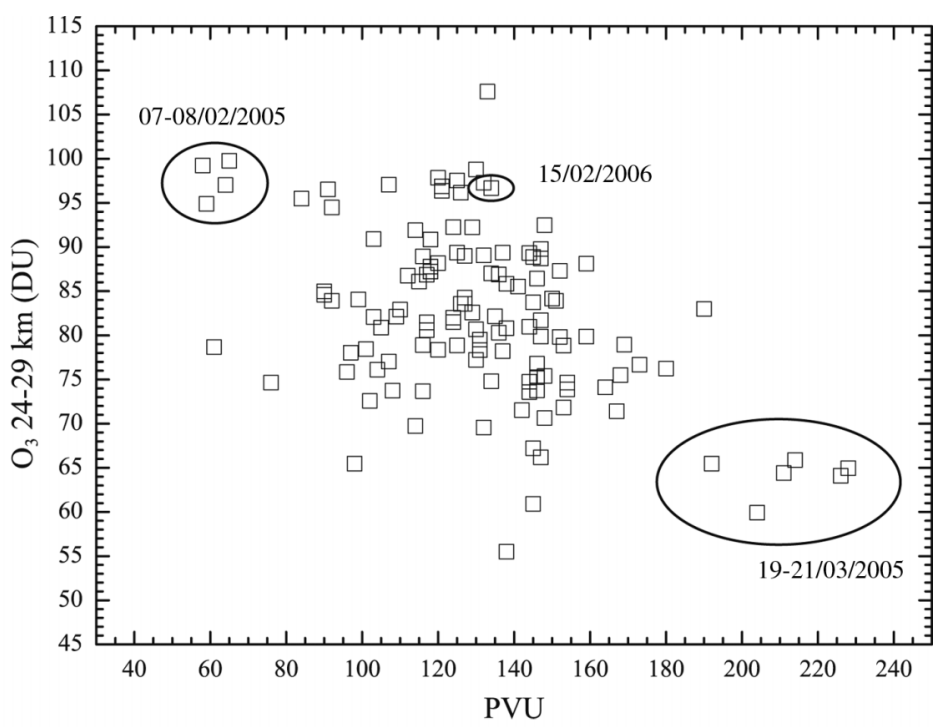

Fig. 9. Correlation scatterplot between Testa Grigia GBMS column density values between 24 and $29 \mathrm{~km}$ altitude (in Dobson Units) and averages of Potential Vorticity values along 15-day back trajectories at $650 \mathrm{~K}(\sim 27$ $\mathrm{km}$ altitude). Specific dates are circled and indicated in the figure (see text for details). PVU stands for Potential Vorticity Unit with $1 \mathrm{PVU}=10^{-6} \mathrm{Km}^{2} \mathrm{~kg}^{-1} \mathrm{~s}^{-1}$.
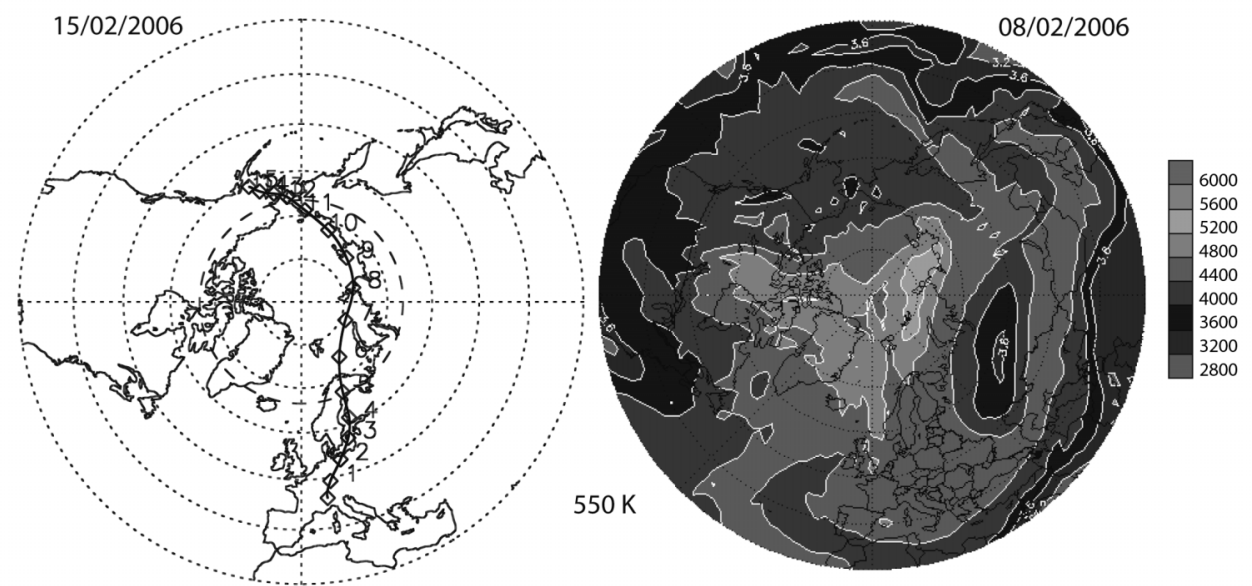

Fig. 10. NASA/GSFC 15-day back trajectory at $550 \mathrm{~K}(\sim 25 \mathrm{~km}$ altitude $)$ arriving over Testa Grigia on 15 February, 2006 (left) and an ozone mixing ratio contour map at $550 \mathrm{~K}$ for 8 February, 2006 (right) produced by the REPROBUS model calculations downloaded from <http://ether.ipsl.jussieu.fr/etherTypo $>$.

and the PV at a potential temperature of $650 \mathrm{~K}$ $(\sim 27 \mathrm{~km})$. This analysis was aimed at studying the variations of $\mathrm{O}_{3}$ in the middle stratosphere in connection with a parameter related to stratospheric dynamics that could discriminate air masses belonging to the polar vortex (high PV) against air masses advected over TG from the tropical region (low PV). Although the 
overall correlation is poor, we circled two sets of datapoints which show characteristic values of the tropical lower stratosphere (7-8/02/2005) and of polar vortex air (19-21/03/2005). Their different origin was confirmed by air parcel trajectories at $650 \mathrm{~K}$. As for all the data points in between the two extreme conditions, the lack of correlation is due to the very complex mixing processes that characterize stratospheric air at mid-latitudes. Even if a certain air mass observed at mid-latitudes is clearly advected from the Arctic region, this parcel can have very different characteristics depending on whether it carries air from the Aleutian high or from the polar vortex. This is the case, for example, for February 2006 (February 15 is also circled in fig. 9 , February 14 is the nearby point with the largest $\mathrm{O}_{3}$ content, $\left.\sim 108 \mathrm{DU}\right)$ when air masses reaching TG appear to have been advected from a region where a large gradient in $\mathrm{O}_{3}$ vmr exists, as shown in fig. 10. Sorting datapoints within the correlation shown in fig. 9 in order to quantitatively pin down the origin of the air masses sampled is therefore very difficult. The use of atmospheric tracers such as $\mathrm{N}_{2} \mathrm{O}$, also measured by the GBMS, will be implemented in a future work in order to better characterize lower stratospheric dynamics.

\section{Acknowledgements}

This research was carried out with a grant from Italy's National Program for Antarctic Research (PNRA) and with support from MIUR PRIN 2005, project \#2005025202/Area 02. We made considerable use of the NASA-Goddard «Automailer» system in tracking the dynamics of stratospheric air over Testa Grigia, and we thank Leslie Lait, Paul Newman, and Mark Schoeberl for providing this service for external users. We thank Meteoswiss for making available their ozone sonde measurements from Payerne through the Network for the Detection of Atmospheric Composition Change (NDACC) website. We are indebted to Michael Pezzopane and Giorgio di Sarra for helpful comments on the original manuscript and to the Istituto di Fisica dello Spazio Interplanetario (INAF/IFSI) and the Centro Nazionale di Meteorologia e
Climatologia Aeronautica (CNMA) for logistic support at Testa Grigia.

\section{REFERENCES}

BraAthen, G. (2006): Joint WMO/EU Arctic Ozone Bulletin, Winter/Spring Summary (World Meteorological Organization/European Ozone Research Coordinating Unit), Tech. Rep. 2006-1 (available on line at: <http://www.wmo.int/pages/prog/arep/gaw/ozone/in dex.html >).

Cheng, D., R.L. De Zafra and C. Trimble (1996): Millimeter wave spectroscopic measurements over the South Pole, 2. An 11-month cycle of stratospheric ozone observations during 1993-1994, J. Geophys. Res., 101, 67816793.

DE ZAFRA, R.L. (1995): The ground-based measurements of stratospheric trace gases using quantitative millimeter wave emission spectroscopy, in 'Diagnostic Tools in Atmospheric Physics', Proceedings of the International School of Physics «Enrico Fermi», edited by G. FIOcCO and G. VISCONTI (Elsevier, New York), 124, 23-54.

De ZAFrA, R.L. and G. MusCARI (2004): CO as an important high-altitude tracer of dynamics in the polar stratosphere and mesosphere, J. Geophys. Res., 109 (D06105), doi: 10.1029/2003JD004099.

De Zafra, R. L., V. Chan, S. Crewell, C. Trimble and J.M. REEVES (1997): Millimeter wave spectroscopic measurements over the South Pole, 3. The behavior of stratospheric nitric acid through polar fall, winter, and spring, J. Geophys. Res., 102, 1399-1410.

Dobson, G.M.B., D.N. Harrison and J. Lawrence (1929): Measurements of the amount of ozone in the earth's atmosphere and its relation to other geophysical conditions, Proc. R. Soc. London A, 122, 456-486.

Hood, L.L., B.E. Soukharev, M. Fromm and J.P. McCorMACK (2001): Origin of extreme ozone minima at middle to high northern latitudes, J. Geophys. Res., 106 (D18), 20,925-20,940.

Jeannet, P., R. Stübi, G. LeVrat, P. Viatte and J. Staehelin (2007): Ozone balloon soundings at Payerne (Switzerland): reevaluation of the time series 1967-2002 and trend analysis, J. Geophys. Res., 112 (D11302), doi: 10.1029/2005JD006862.

Keckhut, P., C. David, M. Marchand, S. Bekki, J. JumeLET and A. HAUCHECORNE (2007): Observation of Polar Stratospheric Clouds down to the Mediterranean coast, Atmos. Chem. Phys. Discuss., 7, 6557-6572.

KeIL, M., D.R. JACKSON and M. C. HoRT (2007): The January 2006 low ozone event over the U.K., Atmos. Chem. Phys., 7, 961-972.

Koch, G., H. Wernli, J. Staehelin and T. Peter (2002): A lagrangian analysis of stratospheric ozone variability and long-term trends above Payerne (Switzerland) during 1970-2001, J. Geophys. Res., 107 (D19), 4373, doi: $10.1029 / 2001 \mathrm{jd} 001550$.

Koch, G., H. Wernli, C. Schwierz, J. Staehelin and T. Peter (2005): A composite study on the structure and formation of ozone miniholes and minihighs over Central Europe, Geophys. Res. Lett., 32 (L12810), doi: 10.1029/2004GL022062. 
Manney, G.L., W.H. Daffer, K.B. Strawbridge, K.A. WALKER, C.D. BoOne, P.F. BeRnATH, T. KeRZENMACHER, M.J. Schwartz, K. Strong, R.J. SicA, K. KrÜger, H.C. Pumphrey, L. Froidevaux, A. Lambert, M.L. SANTeE, N.J. Livesey, E.E. REMSBERG, M.G. MLynCZAK and J.R. Russell III (2007): The high Arctic in extreme winters: vortex, temperature, and MLS and ACE-FTS trace gas evolution, Atmos. Chem. Phys. Discuss., 7, 10235-10285.

Muscari, G., A. G. Di SARra, R. L. De Zafra, F. LuCCi, F. BAORdo, F. ANGELINI and G. FiocCo (2007): Middle atmospheric $\mathrm{O}_{3}, \mathrm{CO}, \mathrm{N}_{2} \mathrm{O}, \mathrm{HNO}_{3}$, and temperature profiles during the warm Arctic winter 2001-2002, J. Geophys. Res., 112 (D14304), doi: 10.1029/2006JD007849.

PARrish, A., R.L. De ZAFra, P.M. SOlOMON and J.W. BARRETT (1988): A ground-based technique for millimeter wave spectroscopic observations of stratospheric trace constituents, Radio Sci., 23, 106-118.

SCHOEBERL, M.R. and L.C. SPARLING (1995): Trajectory modelling, in 'Diagnostic Tools in Atmospheric Physics', Proceedings of the International School of Physics «En- rico Fermi», edited by G. FIOCCO and G. VISCONTI (Elsevier, New York), 124, 289-305.

Stick, C., K. Krüger, N.H. Schade, H. Sandmann and A. MACKE (2006): Episode of unusual high solar ultraviolet radiation over Central Europe due to dynamical reduced total ozone in May 2005, Atmos. Chem. Phys., 6, 1771-1776.

Teitelbaum, H., C. Basdevant, A. Hertzog and N. SeMANE (2003): Comment on «A Lagrangian analysis of stratospheric ozone variability and long-term trends above Payerne (Switzerland) during 1970-2001» by G. Koch, H. Wernli, J. Staelin and T. Peter, J. Geophys. Res., 108 (D21), 4674, doi: 10.1029/2003JD003687.

Twomey, S., B. Herman and R. RABINOFF (1977): An extension of the Chahine method of inverting the radiative transfer equation, J. Atmos. Sci., 34 (7), 1085-1090.

(received September 14, 2007; accepted October 29, 2007) 